\title{
A Convolutional Neural Network Approach for Classification of LPWAN Technologies: Sigfox, LoRA and IEEE 802.15.4g
}

\author{
Adnan Shahid*, Jaron Fontaine*, Miguel Camelo ${ }^{\dagger}$, Jetmir Haxhibeqiri*, Martijn Saelens*, Zaheer Khan ${ }^{\ddagger}$, \\ Ingrid Moerman*, Eli De Poorter* \\ * imec - IDLab, Department of Information Technology at Ghent University \\ * IDLab, Department of Mathematics and Computer Science, University of Antwerp - imec \\ $\ddagger$ CWC, University of Oulu, Finland \\ Email: adnan.shahid@ugent.be
}

\begin{abstract}
This paper presents a Convolutional Neural Network (CNN) approach for classification of low power wide area network (LPWAN) technologies such as Sigfox, LoRA and IEEE 802.15.4g. Since the technologies operate in unlicensed sub-GHz bands, their transmissions can interfere with each other and significantly degrade their performance. This situation further intensifies when the network density increases which will be the case of future LPWANs. In this regard, it becomes essential to classify coexisting technologies so that the impact of interference can be minimized by making optimal spectrum decisions. State-of-the-art technology classification approaches use signal processing approaches for solving the task. However, such techniques are not scalable and require domain-expertise knowledge for developing new rules for each new technology. On the contrary, we present a CNN approach for classification which requires limited domain-expertise knowledge, and it can be scalable to any number of wireless technologies. We present and compare two CNN based classifiers named CNN based on in-phase and quadrature (IQ) and CNN based on Fast Fourier Transform (FFT). The results illustrate that CNN based on IQ achieves classification accuracy close to $97 \%$ similar to $\mathrm{CNN}$ based on FFT and thus, avoiding the need for performing FFT.

Index Terms-Convolutional Neural Networks, technology classification, interference, spectrum manager, coexistence
\end{abstract}

\section{INTRODUCTION}

Wireless networks have become extremely ubiquitous in today's modern world. This stems from the explosive growth of the Internet of Thing (IoT) technologies which led to a wide range of application areas such as security, tracking, agriculture, smart metering, smart cities, and smart homes [1]. According to a report from Cisco, there will be around 500 billion IoT devices connected to the internet by 2030 [2]. In order to connect such a range of devices, recently a number of IoT technologies are developed which collectively are termed as low power wide area network (LPWAN).

In the IoT world, the following LPWAN technologies: Sigfox [3] and LoRA [4] have received significant popularity. In addition, the Institute of Electrical and Electronics Engineers (IEEE) has also proposed an amendment for IEEE 802.15.4, i.e., IEEE $802.15 .4 \mathrm{~g}$, which finds its applications in smart metering utility networks [5]. These three technologies operate in unlicensed sub-GHz bands, typically $868 \mathrm{MHz}$ in Europe and $915 \mathrm{MHz}$ in North America. In this work, we focus on the operation of the three technologies in Europe, where a duty cycle limitation of $1 \%$ is imposed. However, the proposition can be applied to any geographical region.

Because the technologies operate in similar frequency bands, their transmission can cause interference with each other and result in degraded signal-to-noise-plus-interference ratio (SINR) performance. Thus, it becomes essential to extract spectrum occupancy information and classify operating technologies so that the probability of successful communication can be enhanced. This set of information brings Cognitive Radio (CR) capability which aids in making optimal spectrum decisions with the concern of minimizing the impact of interference. Within the context of LPWAN operation in Europe, it is assumed there is negligible interference due to the following two reasons: a) the duty cycle limitation and b) the number of messages that an IoT device can send is typically low. However, as the network density increases due to the surge of IoT applications, these assumptions are no longer applicable.

In order to solve the problems, a wireless spectrum monitoring network is required which scans over frequency, time, and space in unlicensed sub-GHz bands. For this, in this paper, we propose a spectrum manager framework whose job is to provide spectrum occupancy and technologies classification information to the participating LPWANs so that the impact interference can be minimized by better managing the spectrum.

Traditionally, technology classification is done by rulebased approaches such as energy-detection, received signal strength indicator (RSSI), etc., but the main problem is that they cannot work when multiple coexisting technologies are considered. For such a case, advanced classification algorithms are required such as cyclostationary feature detection [6]; however, it incurs huge computational complexity. In recent years, there has been a rapid improvement in Neural Network (NN) architectures and optimization algorithms which collectively are termed as deep learning (DL). Particularly, Con- 
volutional Neural Networks (CNNs) have shown tremendous performance in various domains such as image classification [7], modulation classification [8], etc. However, most of the works on technology classification focus on $2.4 \mathrm{GHz}$ instead of sub-GHz band in which technologies have peculiar characteristics such as narrow bandwidths, long packet durations, different modulation schemes, etc. Due to the fact that LPWAN technologies have unique characteristics and the works on technology classification cannot be directly applied, we aim to present a classifier for classification of the following LPWAN technologies: Sigfox, LoRA and IEEE 802.15.4.

The main contributions of our work are twofold. First, we propose a spectrum manager framework for LPWAN which can take optimal spectrum decisions with the concern of minimizing the impact of interference. Second, we propose two CNN based classifiers: a) CNN based on IQ and b) CNN based on FFT of the IQ samples for classification of technologies such as Sigox, LoRA, and IEEE 802.15.4g. The two classifiers are proposed to have a trade-off analysis in different noise conditions.

The remainder of the paper is organized as follows. Section II presents the related work generally in the field of wireless technology classification. In Section III, we provide a description of the spectrum manager framework. Section IV includes a description regarding the dataset generation and description. Section V describes the architecture and implementation details of the proposed classifiers. Section VI presents the results and analyze the classifiers in various ways.

\section{RELATED WORK}

In this section, we divide the related work on technology classification in two parts: a) rule-based approaches and b) deep learning approaches.

Bouzegzi et al. [9] present an algorithm that classifies wireless technologies such as WiMAX, WiFi and DVB-T based on the inter-carrier spacing in orthogonal frequency-division multiplexing (OFDM) of each technology. The algorithm estimates the inter-carrier spacing based on the maximum-likelihood principle. However, the algorithm only works by estimating the inter-carrier spacing which makes it unsuitable for classifying a technology which does not use OFDM. In addition, for each new technology new hand-crafted rules have to be determined which requires domain-expertise knowledge. Previous work [10] focuses on classification of technologies such as LTE, WiFi and DVB-T based on the statistical distribution of RSSI. Similarly, it cannot be extended to new technologies as it requires RSSI distribution of the new ones. Al-Habashna et al. [11] propose an algorithm for classification of LTE and WiMAX technologies based on their second-order cyclostationary properties. The scheme does not require carrier, waveform, and symbol timing information, while in addition, it provides immunity against phase, frequency, and time offsets. However, it incurs huge computation complexity which makes it not suitable for real systems. Using field programmable gate arrays (FPGA), the work in [12] designed and implemented a real-time environment sensing device which can classify between radar and WiFi type signals. However, there are marked differences with our work: a) it focuses on $3.5 \mathrm{GHz}$ and $5 \mathrm{GHz}$ spectrum bands and b) it uses energy detection approaches. Based on the limitations of the rule-based related works, we aim to present an approach which is less complex, scalable, and requires limited domain-expertise knowledge.

Due to the recent advancement in DL, there exist some works on technology classification that exploit time, frequency, and time-frequency features. Schmidt et al. [13] present a CNN based approach for classification of various configurations of technologies such as IEEE $802.11 \mathrm{~b} / \mathrm{g}$, IEEE 802.15.4 and IEEE 802.15.1 operating in $2.4 \mathrm{GHz}$ ISM band using IQ samples. In previous work [14], the authors propose a CNN approach for classification of different variants of technologies (LTE and WiFI) and provide a mechanism so that the two technologies can coexist in a fair manner. All the works focus on classification of technologies in $2.4 \mathrm{GHz}$ industrial, scientific and medical (ISM) and the propositions cannot be directly applicable to LPWAN technologies because of their different characteristics such as long packet durations, narrow bandwidths, and different modulation schemes.

According to the best of our knowledge, classification of LPWAN technologies in sub-GHz unlicensed bands using DL has not been investigated so far. This work is an extension of our recently accepted demo paper [15] where we demonstrate classification of different signal classes of Sigfox, LoRA, IEEE 802.15.4g and Noise. Following are the extensions of our work: a) detailed description of the spectrum manager framework, b) inclusion of the interference signal class, and c) detailed analysis of the two CNN classifiers in different channel conditions per each signal class.

\section{PROPOSED SPECTRUM MANAGER FRAMEWORK}

We propose a spectrum manager framework for classification of LPWAN technologies Sigfox, LoRA and IEEE 802.15.4g as shown in Figure 1. The main goal of the spectrum manager is to classify the technologies and make efficient spectrum decisions for minimizing the impact of interference. In order to achieve this, the following tasks are proposed to be performed in the listed order: 1) offline training of $\mathrm{CNN}$, 2) classify technologies using the trained model, 3) create radio environment maps, and 4) make spectrum decisions. In the first step, offline training of a CNN is carried out with a labeled dataset which comprises of IQ samples of the technologies. In order to have a generalized and robust classifier, all possible configurations such as power levels, modulation schemes, traffic types, etc., of the technologies should be taken into account. In this work, we consider ten signal classes of the technologies (Sigfox, LoRA, and IEEE 802.15.4g): 1 signal class of Sigfox which corresponds to transmissions on all the 400 Sigfox channels, six signal classes of LoRA with spreading factors (SFs) = 7, 8, 9, 10, 11 and 12, 1 class of IEEE $802.15 .4 \mathrm{~g}, 1$ class of interference, and 1 class of noise. The detailed description of the signal classes is given in Section IV. In the second step, the trained model is used for classification of the ten signal classes. In the third 
step, radio environmental maps (REMs) are created based on frequency-domain analysis of the technology classification results. Finally, in the fourth step, based on the spectrum occupancy information extracted, spectrum decisions are made and are notified to the participating LPWANs. This spectrum decision information helps in changing operating channels and power levels of the technologies so that fair co-existence can be realized.

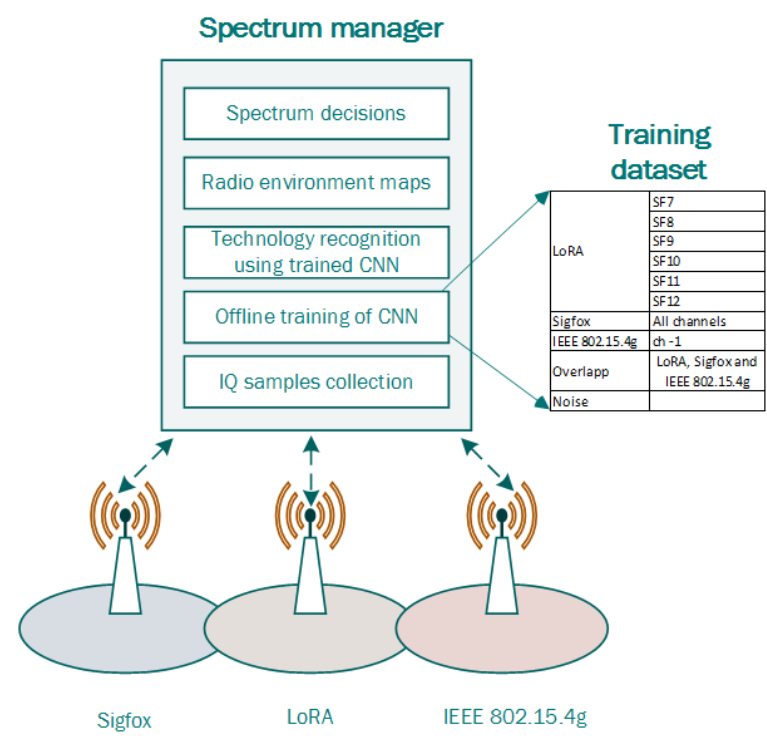

Fig. 1: Proposed spectrum manager framework for classification of LPWAN technologies using CNN.

\section{DATASET GENERATION AND DESCRIPTION}

The hardware setup that we used for collecting IQ samples from the ten signal classes is shown in Figure 2. The setup comprises of three transmitters (that correspond to Sigfox, LoRA and IEEE 802.15.4g), a B200mini universal software radio peripheral (USRP), and a microcontroller. The USRP was used for capturing IQ samples with a sampling rate of $1 \mathrm{M}$ samples/sec, and the rate was selected by taking into account the operating bandwidths of the three technologies. The microcontroller was used to precisely schedule transmissions from the three transmitters and specifically, it was used for creating the interference signal class. As mentioned before, the ten signal classes include one signal class of Sigfox, six signal classes of LoRA, one signal class of IEEE 802.15.4g, one signal class of interference, and one signal class of noise. For each signal class, IQ samples were captured for a duration of $120 \mathrm{sec}(120,000,000$ samples for each signal class) and later on, they were processed for making them compatible with the CNN.

For the Sigfox signal class, we carried out one measurement campaign by making the Sigfox transmitter to operate randomly on the 360 Sigfox channels such that $\left\{c_{1}, c_{2}, \ldots, c_{180}, c_{220}, \ldots, c_{400}\right\}$ with each of bandwidth 100 Hz. For the transmitter, we used a Sigfox TD1207 device

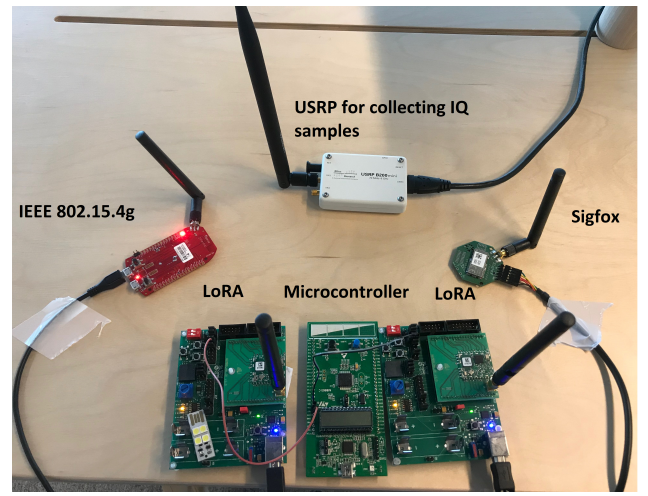

Fig. 2: Dataset generation setup.

and its 'Send RF Test message' mode was used via an AT command to operate on all the 360 channels randomly.

For the six LoRA signal classes, we performed three measurement campaigns. In each campaign, two LoRA transmitters were used with one operating on one SF while the other operating on other SF. In order to avoid overlap, we carefully programmed the nodes triggered by the microcontroller so that each SF signal class did not overlap with each other. From the setup, the packet duration (in sec) and the symbol duration (in ms) of the LoRA transmissions with SFs 7, 8, 9, 10, 11 and 12 are represented as $0.0698,0.12339,0.214029,0.428637$, 0.8564 and 1.715 , respectively and $1.02,2.05,4.1,8.19,16.38$ and 32.77, respectively. The LoRA transmissions of the six signal classes were operated at the center frequency of 868.1 $\mathrm{MHz}$ with a bandwidth of $125 \mathrm{KHz}$.

For the IEEE $802.15 \mathrm{~g}$ signal class, we carried out one measurement campaign to capture IQ samples from the transmitter operating at the center frequency and bandwidth of $868.1 \mathrm{MHz}$ and $600 \mathrm{KHz}$, respectively. The transmitter was programmed such that the packet duration, the symbol duration, and the supported data rate was $0.0162,0.02 \mathrm{~ms}$ and $50 \mathrm{kbps}$, respectively. Furthermore, the modulation scheme of binary frequency shift keying (BFSK) was used.

For the interference signal class, we used the microcontroller to create a setup for having the following interference cases: a) Sigfox and LoRA, b) Sigfox and IEEE 802.15.4.g, c) LoRA and IEEE $802.15 .4 \mathrm{~g}$, and d) all the three technologies. For the sake of simplicity, we combined all the interference cases and categorized them as the single interference signal class. Furthermore, we consider only cross-tier interference (interference between different technologies) and leave cotier (interference between the same technology) for future investigations.

For the noise signal class, we processed all the IQ samples of the nine signal classes described above and based on an energy detection mechanism, IQ samples that correspond to the noise class were extracted.

A visualization of the dataset of four signal classes out of ten is shown in Figure 3 and Figure 4. Since in this work we propose two CNN approaches for technology classification: a) CNN based on IQ and b) CNN based on FFT, the data is 

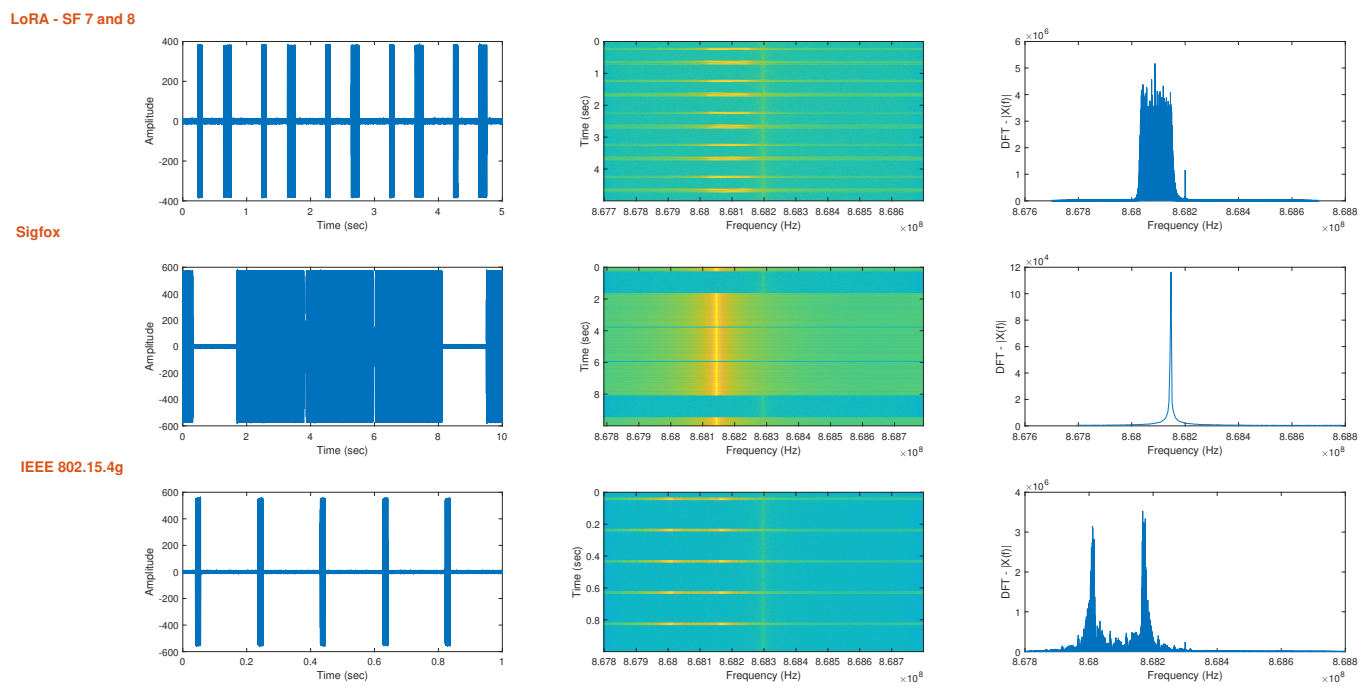

Fig. 3: Dataset visualization of LoRA - SF 7 and 8, Sigfox and IEEE 802.15.4g at SNR $=35 \mathrm{~dB}$.
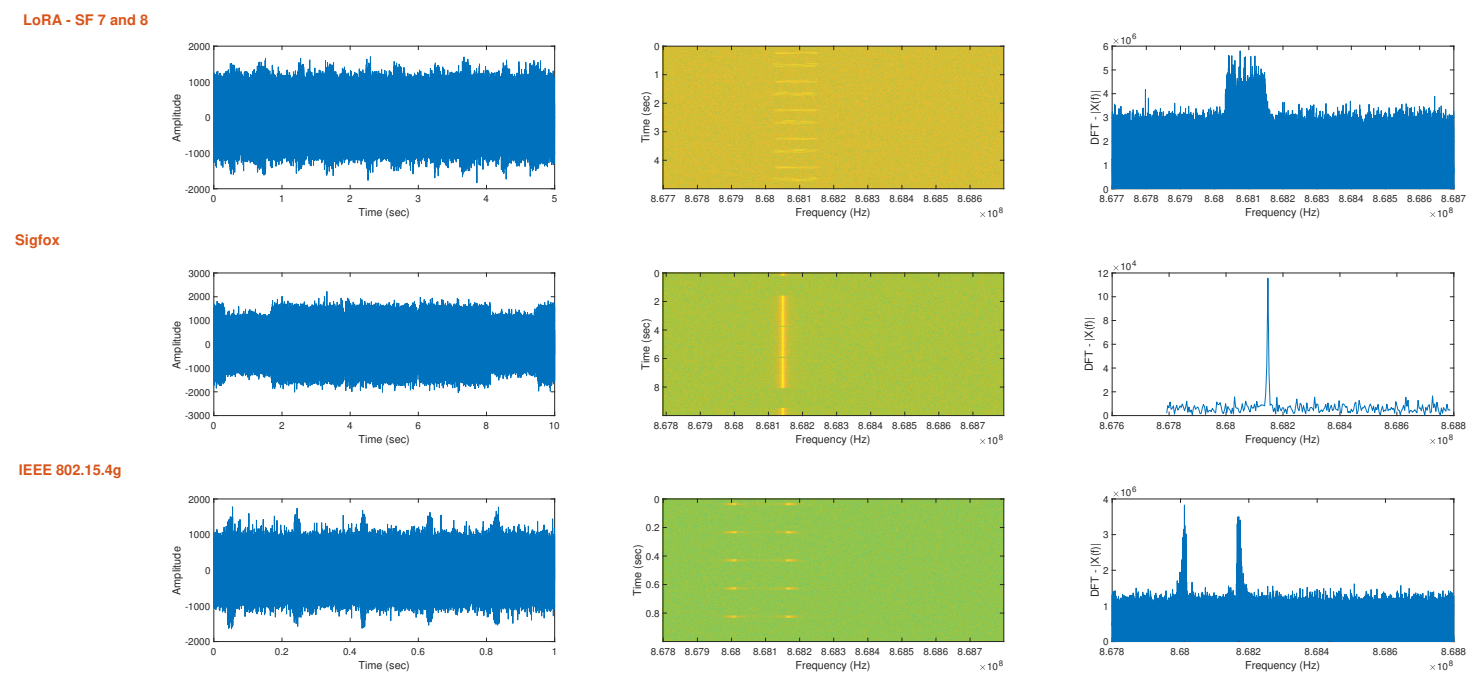

Fig. 4: Dataset visualization of LoRA - SF 7 and 8, Sigfox and IEEE 802.15.4g at SNR $=-10 \mathrm{~dB}$.

presented in the form of time-domain and frequency-domain representations for signal-to-noise ratio $(\mathrm{SNR})=-10 \mathrm{~dB}$ and 35 $\mathrm{dB}$, respectively. The reason for selecting the SNR values of 35 $\mathrm{dB}$ and $-10 \mathrm{~dB}$ is to show how the signal classes look like when noise is added after the IQ samples collection. Specifically, at $-10 \mathrm{~dB}$ all the signal classes in the time-domain (the first column of Figure 3 and Figure 4) are submerged with noise, but at the same SNR, the frequency-domain (the third column of Figure 3 and Figure 4) representation still have distinct features that can be exploited by CNNs.

\section{Convolutional Neural Network Design}

In this section, we propose a CNN architecture which is used for both the classifiers: a) CNN based on IQ and b) CNN based on FFT, where the former one uses raw IQ samples while the later one uses FFT of the IQ samples. The IQ samples dataset that we acquired from the setup comprises of ten signal classes: one signal class of Sigfox, six signal classes of LoRA, one signal class of IEEE 802.15.4g, one signal class of interference, and one signal class of noise. The goal of the proposed classifiers is to identify those ten signal classes. Furthermore, we consider a use case in which all the technologies operate at the center frequency of $868.1 \mathrm{MHz}$, and we made this choice because all the technologies can operate in the same band in Europe concurrently and potentially interfere with each other. In such a coexisting environment, it becomes necessary to have a spectrum manager that assists 
the participating LPWAN (at the node level or at the gateway level), for minimizing the impact of interference.

\section{A. Time and frequency snapshots}

In order to make the captured IQ samples data compatible with the CNN, the data was divided into sensing snapshots, where each sensing snapshot corresponds to 500 IQ samples or $500 \mu \mathrm{s}$. Primarily, the duration of the sensing shot should be greater than or equal to the minimum symbol duration of all the signal classes under consideration. The symbol duration (in ms) of one signal class of Sigfox is 10, six signal classes of LoRA with $\mathrm{SF}=7,8,9,10,11$ and 12 are 1.02, 2.05, $4.1,8.19,16.38$ and 32.77 , respectively, and one signal class of IEEE $802.15 .4 \mathrm{~g}$ is 0.02 . Therefore, the minimum sensing duration is $32.77 \mathrm{~ms}$ or 32770 IQ samples. However, such a large sensing snapshot increases the complexity of CNN. Instead, we use a sensing shot of duration $0.5 \mathrm{~ms}$ or $500 \mathrm{IQ}$ samples and this also reduces the sensing time.

It has been shown that in emission-based devices frequencydomain features outperform than their time-domain equivalents [16]. Inspired from this we carried out a comparison of the proposed $\mathrm{CNN}$ based on IQ and $\mathrm{CNN}$ based on FFT approaches. For the CNN based on FFT, each sensing snapshot of 500 IQ samples was transformed into its equivalent frequency-domain using FFT.

\section{B. Network Structure}

Figure 5 shows the CNN structure for the proposed classifiers and is based on [8]. We have also used a similar structure for the classification of different Long Term Evolution (LTE) and WiFi signal classes [14]. The structure comprises of three convolutional and pooling layers followed by two fully connected layers. The number of neurons in each layer and the convolutional filter sizes are shown in Figure 5.

For both the approaches, the input matrices are of similar dimension and they correspond to either IQ samples or FFT values of the IQ samples. Mathematically, the model inputs can be written as:

$$
X_{e}^{I Q / F F T}=\left[\begin{array}{c}
x_{e, j}^{I}, \ldots, x_{e, M}^{I} \\
x_{e, j}^{Q}, \ldots, x_{e, M}^{Q}
\end{array}\right], \forall e \in[1, N], j \in[1, M],
$$

where $e$ is the sensing snapshot index, $N$ is the total number of sensing snapshots, and $M$ is the number of I and Q samples in each $e$ th sensing snapshot. In our case, $N=200,000$ and $M=500$.

Primarily, the extraction of features is happened by convolutional and pooling layers and are the core part of the proposed $\mathrm{CNN}$ architecture. In the proposed architecture, the output of first convolutional and pooling layers can be written as:

$$
\nabla_{1}=\Pi\left(\alpha_{1}\left(W_{1, f} * X_{e}^{I Q / F F T}+b_{1, f}\right)\right), \forall f \in\left[1, F_{1}\right], e \in[1, N],
$$

where $\Pi$ represents the pooling function, $F_{1}$ represents the number of convolutional filters in the 1 st layer, $\alpha_{1}$ corresponds to the activation function used in the 1 st layer, and $W_{1, f}$ and $b_{1, f}$ are the weights and the bias values, respectively, utilized in the 1st layer. The activation function provides a non-linear mapping between the inputs and the outputs and thus, enables CNN to learn complex and complicated features. Here, we used, ReLu as an activation function and it can be expressed as:

$$
\alpha(x)= \begin{cases}x & \text { if } x>0 \\ 0 & \text { otherwise }\end{cases}
$$

Furthermore, the output of the $l$ th convolutional and pooling layers can also be written as:

$$
\begin{aligned}
& \nabla_{l}=\Pi\left(\alpha_{l}\left(W_{l, f} * \nabla_{l-1}+b_{l, f}\right)\right), \\
& \quad \forall f \in\left[1, F_{1}\right], e \in[1, N], l \in[1, L],
\end{aligned}
$$

where $L$ is the total number of layers. The features learned from the convolutional and pooling layers are converted to a dense vector which subsequently used by the two fully connected layers. This flattening processing is represented as:

$$
\nabla^{\text {flat }}=\Gamma\left(\nabla_{L-1}\right)
$$

where $\Gamma$ is a flatting function which converts the final output of the convolutional and pooling layers $\nabla_{L-1}$ to a dense vector $\nabla^{\text {flat }}$. The flattened output is passed through the two fully connected layers and the final output of the model can be written as:

$$
\begin{array}{r}
\hat{O}_{e}=W_{F C}\left(\Gamma\left(\Pi\left(\alpha_{l}\left(W_{l, f} * \nabla_{l-1}+b_{l, f}\right)\right)\right)\right)+b_{F C} \\
\forall f \in\left[1, F_{1}\right], e \in[1, N], l \in[1, L],
\end{array}
$$

where $W_{F C}$ and $b_{F C}$ are weight and bias parameter values of the fully connected layers and $\hat{O}_{e}$ is the predicted score for each signal class per sensing snapshot.

The last fully connected layer of the architecture is the softmax layer with 10 neurons which corresponds to the fact that it is able to classify the 10 signal classes.

\section{Network training and validation}

The following two sensing snapshot matrices: $X^{I Q}$ and $X^{F F T}$ are utilized for training of both the classifiers CNN based on IQ and CNN based on FFT, respectively. The total sensing snapshots of each matrix are of size 200,000, and the number of sensing snapshots per signal class is equally distributed. For training of each classifier, we divided the corresponding matrix for training and validation in a ratio of 60 and 40, respectively, and the whole data was normalized in the range from -1 to 1 . Furthermore, the Adam optimizer [17] was used which gave the best accuracy results. All the default values of the Adam optimizer were used except the learning rate, which value was changed from 0.001 to 0.0001 to obtain better results. In order to have a fine balance between overfitting and underfitting, a batch size of 1024 was used. 


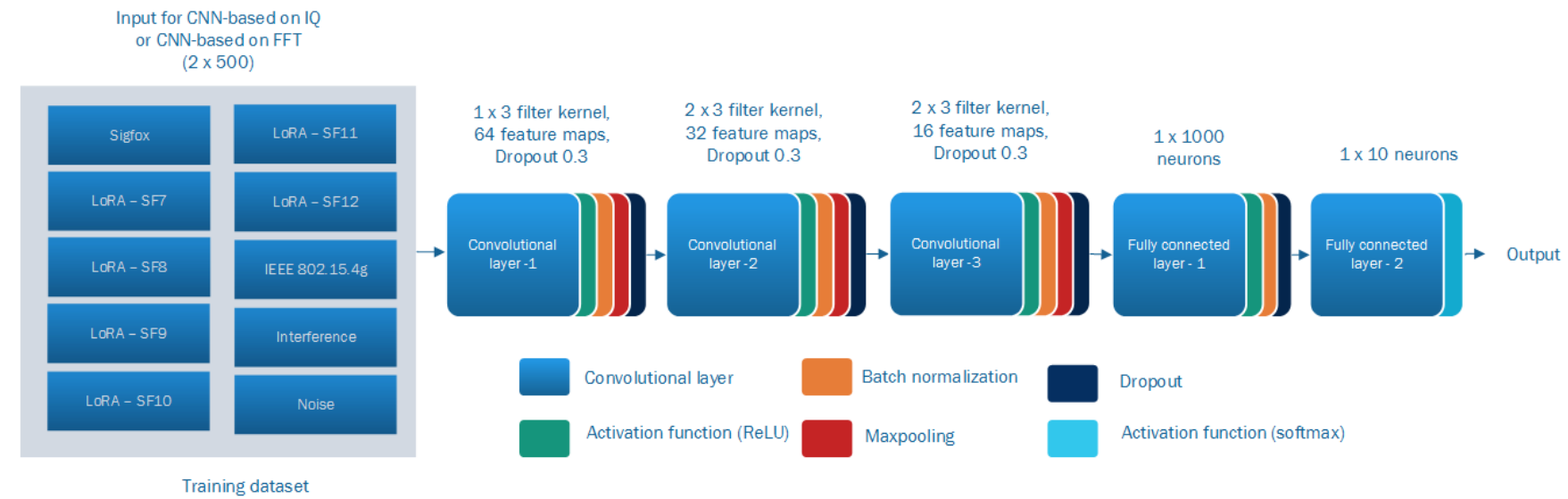

Fig. 5: Proposed architecture for CNN based on IQ and CNN based on FFT.

\section{Implementation}

Both the classifiers were trained and validated on high-end graphics processing units (GPUs) such as NVIDIA GTX 1080 Ti, NVIDIA V100, etc., available in our in-house Virtual Wall [18]. The classifiers were implemented using an abstract level library Keras [19] with Tensorflow [20] as a backend.

\section{RESULTS AND DISCUSSION}

In this section, the comparison of the proposed classifiers is presented in terms of 1) training and validation loss, 2) training and validation accuracy, 3) classification accuracy with respect to SNR, and 4) classification accuracy for each signal class in the form of a confusion matrix.

Figure 6 shows the training and validation loss of the proposed classifiers with respect to the number of epochs. The training loss is defined as an average loss over each batch of the training data while the validation loss is computed subsequently from the trained model at the end of each epoch. In order to have a fine balance between overfitting and underfitting, a dropout of $30 \%$ and regularization by using an L2 regularizer were used, and this is evident from the trend of the training and validation loss curves. Although the loss curves of both the classifiers follow a similar trend, $\mathrm{CNN}$ based on FFT slightly outperforms its IQ-based counterpart. This is because that frequency-domain features are dominant in low noise conditions as compared to time-domain as can be seen in Figure 4.

Figure 7 illustrates the training and validation accuracy achieved during the training phase of the classifiers. The training was done for 200 epochs, and the validation accuracy of CNN based on IQ and CNN based on FFT at this epoch achieve up to $95 \%$ and $97 \%$. This $2 \%$ improvement is due to the presence of better frequency-domain features in the dataset $X^{F F T}$ especially in low noise conditions.

Figure 8 compares the accuracy performance of both the classifiers in terms of SNR. It can be seen that the CNN based on FFT classifier outperforms for low SNRs as compared to its counterpart. This difference is more pronounced at the lowest

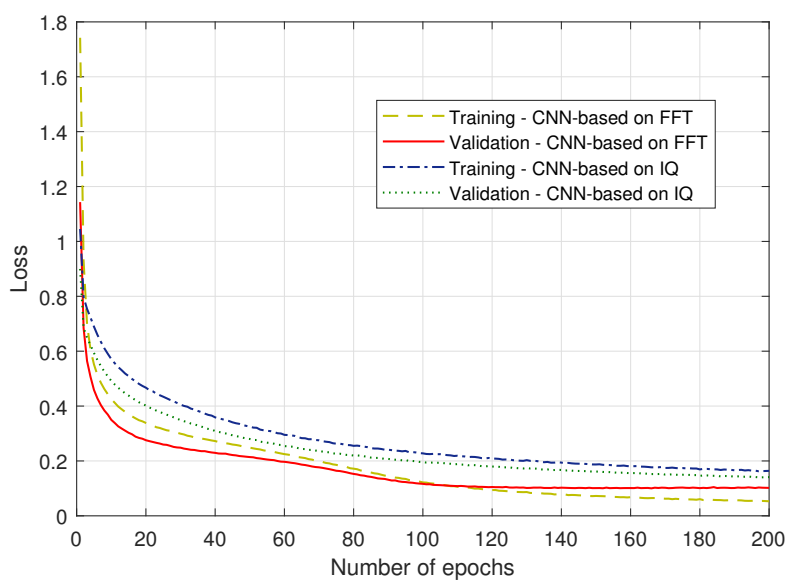

Fig. 6: Training and validation loss.

SNR, i.e., at $-10 \mathrm{~dB}$ and reduces to almost zero at $10 \mathrm{~dB}$. Although CNN based on FFT performs better for low SNRs, it requires further processing for converting IQ samples to frequency-domain while CNN based on IQ does not need any processing.

Figure 9 illustrates the classification accuracy of CNN based on IQ and CNN based on FFT for the 10 signal classes. It can be seen that CNN based on FFT outperforms as compared to its counterpart for classification of all the signal classes for SNR $=-10 \mathrm{~dB}$ and $0 \mathrm{~dB}$. The reason is already described while analyzing Figure 8. If we compare the confusion matrices of Figure 9 (a) and (d), it is evident that CNN based on IQ wrongly identifies all the signal classes as noise and this is due to the fact that there are noise-like features in time-domain representation at SNR of $-10 \mathrm{~dB}$ as shown in the first column of Figure 4. While at a SNR of $35 \mathrm{~dB}$ both the approaches perform similarly. 


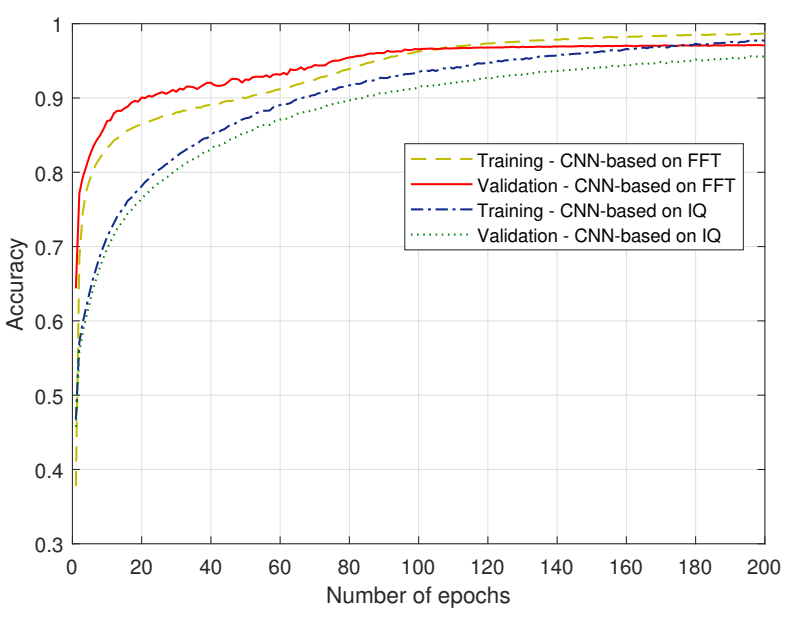

Fig. 7: Training and validation accuracy.

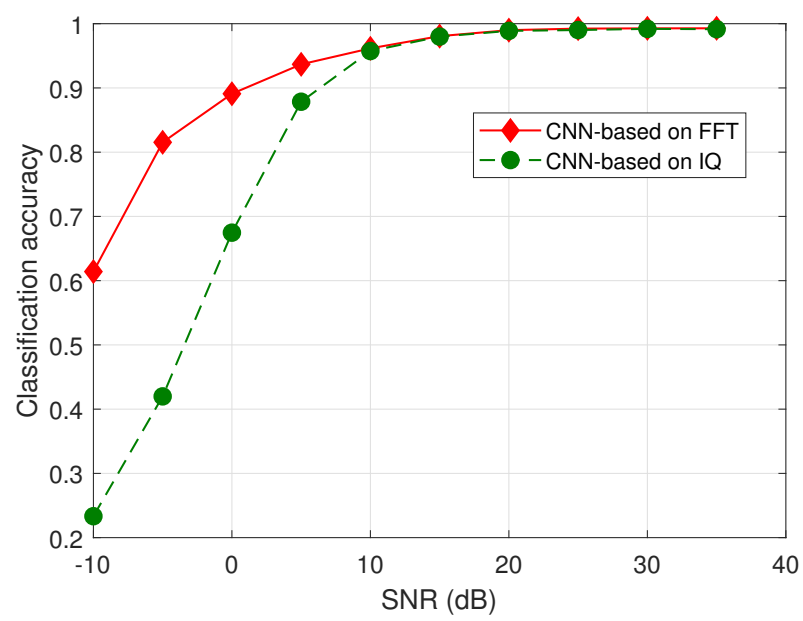

Fig. 8: Classification accracy with respect to SNR.

\section{CONCLUSION}

Most of the LPWAN technologies operate in sub-GHz unlicensed bands. Due to their operation in the unlicensed bands and the increase in IoT network density, optimal spectrum management, and technology classification are of vital importance. Different from state-of-the-art classification approaches which are complex, not scalable, and requires domain-expertise knowledge. Here, we aim to propose a classifier which is less-complex, scalable and does not require domain-expertise knowledge. Particularly, we propose and compare two classifiers for classification of the ten signal classes of the technologies (Sigfox, LoRA and IEEE 802.15.4g): CNN based on IQ and CNN based on FFT. The former requires raw IQ samples, and the later requires FFT of the IQ samples. From the analysis, it is evident that for channel conditions represented by SNR $>10 \mathrm{~dB}$, the classification accuracy of CNN based on IQ is close to CNN based on FFT. Therefore, for such channel conditions, CNN-based on IQ leverages the use of only raw IQ samples and limited domain- expertise knowledge. However, for low SNR conditions, CNN based on FFT outperforms because frequency-domain features are more dominant for such channel conditions.

\section{REFERENCES}

[1] R. Ratasuk, N. Mangalvedhe, and A. Ghosh, "Overview of lte enhancements for cellular iot," in 2015 IEEE 26th annual international symposium on personal, indoor, and mobile radio communications (PIMRC). IEEE, 2015, pp. 2293-2297.

[2] Cisco, [Online] https://www.cisco.com/c/dam/en/us/products/collateral/se/internetof-things/at-a-glance-c45-731471.pdf, [May 16, 2019].

[3] Sigfox, [Online] https://www.sigfox.com, [May 16, 2019]

[4] Lora Alliance, [Online] https://www.lora-alliance.org/, [May 16, 2019].

[5] U. Raza, P. Kulkarni, and M. Sooriyabandara, "Low power wide area networks: An overview," IEEE Communications Surveys \& Tutorials, vol. 19, no. 2, pp. 855-873, 2017.

[6] K. Kim, I. A. Akbar, K. K. Bae, J.-S. Um, C. M. Spooner, and J. H. Reed, "Cyclostationary approaches to signal detection and classification in cognitive radio," in New frontiers in dynamic spectrum access networks, 2007. DySPAN 2007. 2nd IEEE international symposium on. IEEE, 2007, pp. 212-215.

[7] D. Cireşan, U. Meier, and J. Schmidhuber, "Multi-column deep neural networks for image classification," arXiv preprint arXiv:1202.2745, 2012.

[8] T. J. O'Shea, J. Corgan, and T. C. Clancy, "Convolutional radio modulation recognition networks," in International conference on engineering applications of neural networks. Springer, 2016, pp. 213-226.

[9] A. Bouzegzi, P. Ciblat, and P. Jallon, "Maximum likelihood based methods for ofdm intercarrier spacing characterization," in 2008 IEEE 19th International Symposium on Personal, Indoor and Mobile Radio Communications, 2008, pp. 1-5.

[10] W. Liu, M. Kulin, T. Kazaz, A. Shahid, I. Moerman, and E. De Poorter, "Wireless technology recognition based on rssi distribution at subnyquist sampling rate for constrained devices," Sensors, vol. 17, no. 9, p. 2081,2017

[11] A. Al-Habashna, O. A. Dobre, R. Venkatesan, and D. C. Popescu, "Second-order cyclostationarity of mobile wimax and lte ofdm signals and application to spectrum awareness in cognitive radio systems," IEEE Journal of Selected Topics in Signal Processing, vol. 6, no. 1, pp. 26-42, 2012.

[12] Z. Khan, J. J. LehtomÃd'ki, E. Hossain, M. Latva-Aho, and A. Marshall, "An fpga-based implementation of a multifunction environment sensing device for shared access with rotating radars," IEEE Transactions on Instrumentation and Measurement, vol. 67, no. 11, pp. 2561-2578, Nov 2018.

[13] M. Schmidt, D. Block, and U. Meier, "Wireless interference identification with convolutional neural networks," in 2017 IEEE 15th International Conference on Industrial Informatics (INDIN). IEEE, 2017, pp. 180-185.

[14] V. Maglogiannis, A. Shahid, D. Naudts, E. De Poorter, and I. Moerman, "Enhancing the coexistence of lte and wi-fi in unlicensed spectrum through convolutional neural networks," IEEE Access, vol. 7, pp. 28 464-28 477, 2019.

[15] A. Shahid, J. Fontaine, M. Camelo, J. Haxhibeqiri, M. Saelens, Z. Khan, I. Moerman, and E. De Poorter, "Demo abstract: Identification of lpwan technologies using convolutional neural networks," [Online] http://www.ee.oulu.fi/ zaheer/demo2018.pdf, [May 16, 2019].

[16] B. Danev and S. Capkun, "Transient-based identification of wireless sensor nodes," in Proceedings of the 2009 International Conference on Information Processing in Sensor Networks. IEEE Computer Society, 2009 , pp. 25-36.

[17] D. P. Kingma and J. Ba, "Adam: A method for stochastic optimization," arXiv preprint arXiv:1412.6980, 2014.

[18] imec testbed, [Online] https://doc.ilabt.imec.be/ilabt/virtualwall/, [May $16,2019]$.

[19] Keras, [Online] https://keras.io/, [May 16, 2019].

[20] Tensorflow, https://www.tensorflow.org/, [May 16, 2019]. 


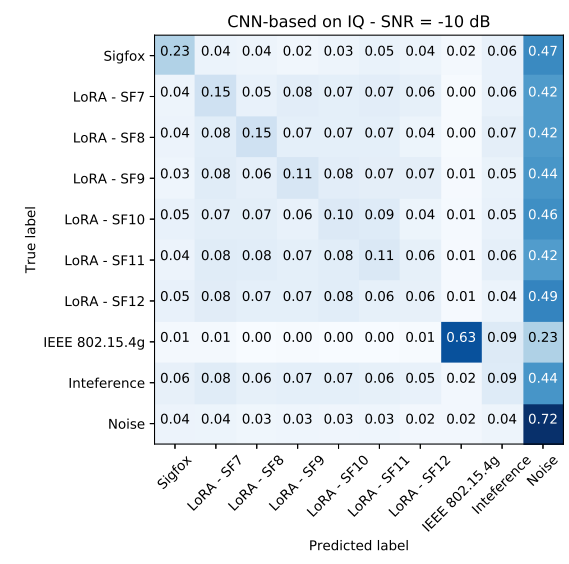

(a)

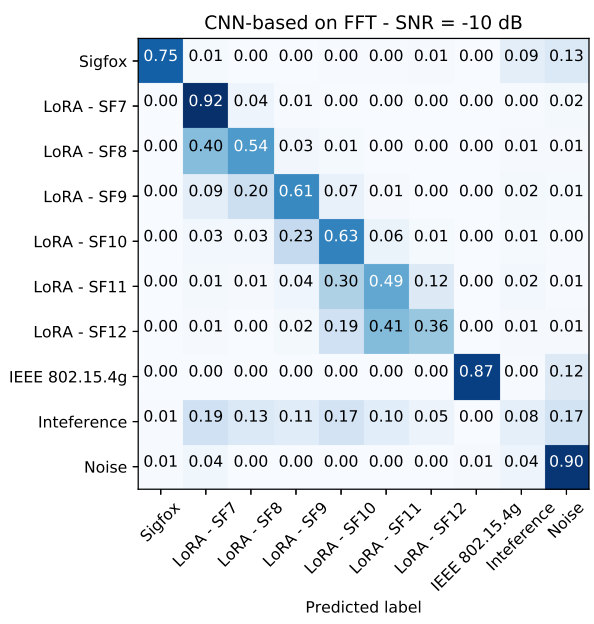

(d)

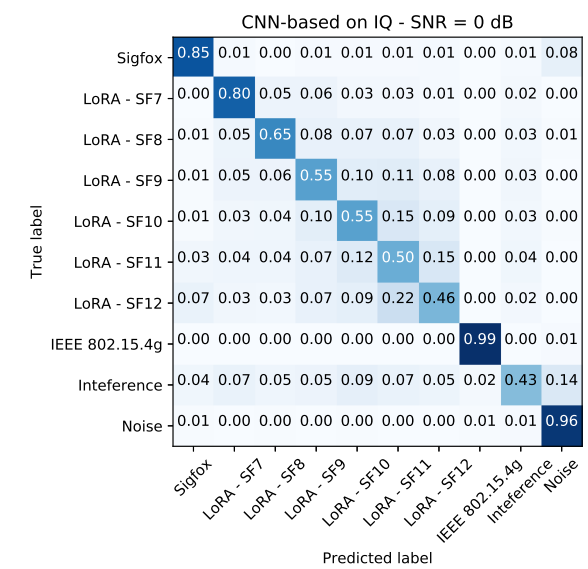

(b)

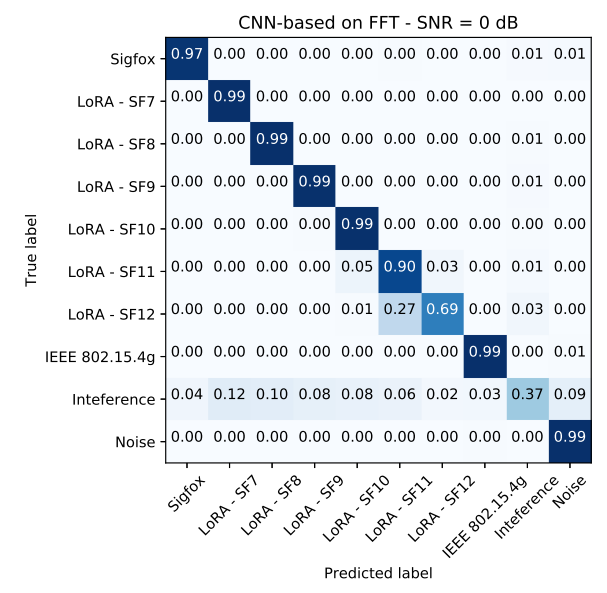

(e)

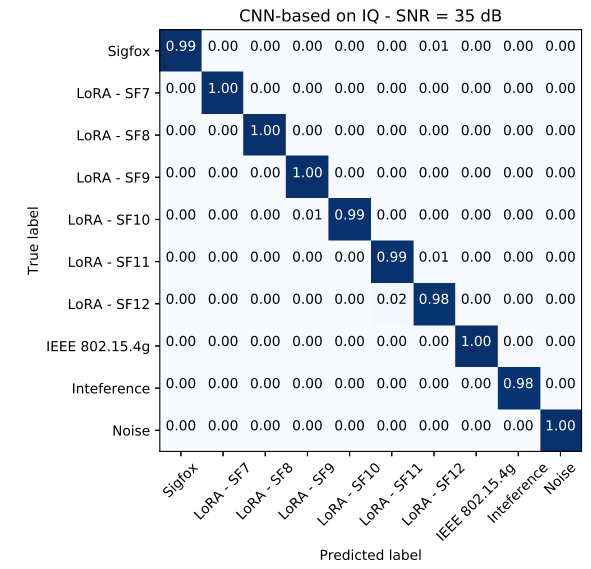

(c)

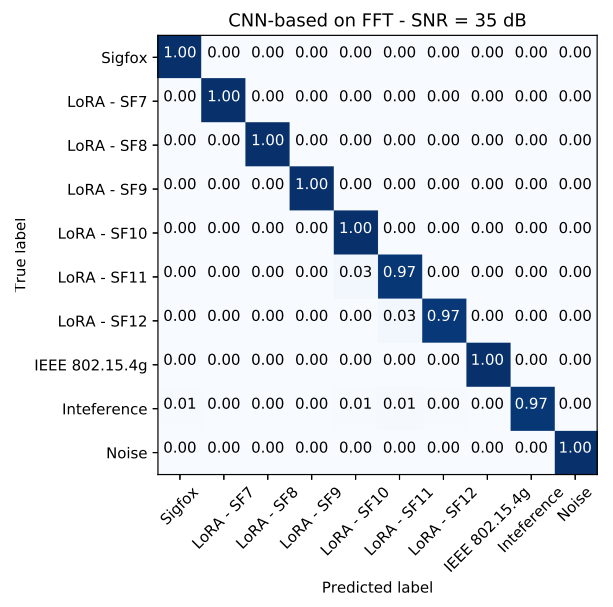

(f)

Fig. 9: Confusion matrices for CNN based on IQ (top row) and CNN based on FFT (bottom row) at SNR $=-10,0$ and 35 dBs. 